\title{
Performance Analysis Of A Concatenated LDPC CODED ALAMOUTI-BASED STBC-OFDM System ON TEXT MEssage Transmission
}

\author{
M. Mesbahul Alam, A.Z.M. Touhidul Islam and S. Enayet Ullah \\ Department of Information and Communication Engineering \\ University of Rajshahi, Rajshai-6205, Bangladesh \\ polash_icedyahoo.com, touhid_ict_rulyahoo.com, enayet67@yahoo.com
}

\begin{abstract}
This paper investigates the impact of concatenation of an interleaved low density parity check (LDPC) code with convolutional channel coding on the performance of the space time block coded (STBC) orthogonal frequency-division multiplexing (OFDM) wireless communication system on text message transmission. The concatenated channel encoded OFDM system deploys various digital modulations (BPSK, QPSK and QAM) over an additative white gaussian noise (AWGN) and other fading (Raleigh and Rician) channels. Computer simulation results on bit error rate (BER) demonstrate that the 1/2-rated convolutional and LDPC channel encoded OFDM system outperforms with BPSK modulation technique as compared to other digital modulation schemes and the system is highly effective to combat inherent interferences under AWGN, Rayleigh and Rician fading channels. The BER performance of the concatenated channel encoded OFDM system with 1/2-rated channel coding is better as compared to 3/4rated channel coding. The transmitted text message is found to have retrieved effectively under noisy and fading situations. It is anticipated from the BER simulations that the performance of the communication system degrades with the increase of noise power.
\end{abstract}

\section{KEYWORDS}

Orthogonal frequency division multiplexing (OFDM), Bit error rate (BER), Low density parity check (LDPC), Space-time block coding (STBC), Convolutional coding.

\section{INTRODUCTION}

The next generation wireless systems are required to have better voice quality and coverage, be more power and bandwidth efficient, and be deployed in diverse environments. The fundamental phenomenon, which makes reliable wireless transmission difficult, is time-varying multipath fading [1]. In most scattering environments, antenna diversity is a practical, effective and, hence, a widely applied technique for reducing the effect of multipath fading. The classical approach is to use multiple antennas at the receiver and perform combining or selection and switching in order to improve the quality of the received signal. The major problem with using the receive diversity approach is the cost, size, and power of the remote units. The use of multiple antennas and radio frequency (RF) chains (or selection and switching circuits) makes the remote units larger and more expensive. As a result, diversity techniques have almost exclusively been applied to base stations to improve their reception quality. A base stations often serves hundreds to thousands of remote units. For this reason, transmit diversity schemes are very attractive.

Recently, multiple antenna techniques have been extensively studied for high rate data transmission and increasing transmission efficiency [2-7]. The space-time block coding (STBC) technique, one of the representative multiple antenna techniques, is most attractive for these DOI : 10.5121/ijcsea.2011.1301 
purposes. STBC, an effective transmit diversity technique, was first proposed by Alamouti [4] for flat fading channel. Alamouti suggested a space time code for two transmit antennas, which provides a diversity gain and has a very simple decoder. On the other hand, the OFDM technique has been widely accepted for the transmission of high rate data due to its robustness to inter-symbol interference. In this context, the STBC-OFDM system may be one of most promising system configurations that can be adopted for $4^{\text {th }}$ generation mobile systems. The combination of STBC and OFDM results in an enhanced system performance in wideband wireless channels [8],[9].

For the sake of further improving the performance, forward error correction (FEC) schemes may be invoked for protecting the subcarriers against frequency selective fading in an OFDM environment. Many error-correcting codes have been applied to OFDM such as convolutional codes, Reed-Solomon codes, Turbo codes [10], and so on. Recently, low-density parity-check (LDPC) codes have attracted much attention particularly in the field of coding theory. LDPC codes were proposed by Gallager in 1962 [11],[12] and are now recognized as good errorcorrecting codes achieving near Shannon limit performance[13]. In order to provide channel coding gain, the family of low density parity check (LDPC) codes has emerged as an attractive alternative to turbo coding [9]. The performance of LDPC codes has been also evaluated on a block fading channel and it has been shown that the LDPC codes achieve a large gain with respect to convolutional codes for large packet length [14]. [11] showed that LDPC codes are effective to improve the error performance of OFDM in multipath environments. It has also been shown in [15] that LDPC based space-time coded OFDM systems are capable of efficiently exploiting the achievable spatial diversity in wireless channel. The concatenation of convolutional and LDPC channel coding is employed in OFDM system for achieving good error rate performance with reasonable complexity.

Throughout this paper, we only consider a $2 \times 1$ system with two transmit and one receive antennas. The STBC-OFDM system is developed by applying Alamouti's representative STBC scheme to the OFDM system for text message transmission. We evaluate the bit error rate (BER) of the STBC-OFDM system with concatenation of outer low-density parity-check and inner convolutional channel coding schemes and compare the BER performance of the system working under each of the three types of digital modulation (BPSK, QPSK and QAM) on the AWGN, Rayleigh and Rician fading channels.

A brief survey of literature in the area relevant to this paper is as follows. J. Ha et al. [16] proposed LDPC code for both Alamouti-OFDM scheme and singular-value decomposition (SVD)-OFDM scheme to provide low BER at a high spectral efficiency and low SNR. Further to enhance system performance M.Y. Alias et al. [17] proposed concatenated LDPC and Turbo coding assisted space-time block coded wireless OFDM system. J. Wu and H-N Lee [18] have shown that channel capacity can be significantly increased by using LDPC coded modulation in multiple-input multiple-output (MIMO) multiple-access systems. The BER performance of a concatenated LDPC encoded OFDM system is studied by M.D. Haque et al. [19] and concluded that the proposed system is very much effective in proper identification and retrieval of transmitted color image in noisy and fading environment. M. M. Hossain et al. [20] also studied the impact of LDPC on the performance of an OFDM system under various digital modulations over an AWGN and other fading channels and they shown that the proposed system with deployment of QAM modulation is highly effective to combat inherent interferences and to retrieve the transmitted black and white image properly under noisy and fading situations.

\section{SYSTEM MODEL}

The architecture of our STBC-OFDM system for text message transmission that utilizes a concatenated LDPC scheme with $n_{T}=2$ transmit and $n_{R}=1$ receive antennas as shown in 
Figure 1. At the transmitter, a text messages "Information Technology is a very important sector in modern world. It is a computer based education." is taken as input. The Text message is converted into binary data of length 696 bits. The binary bits are encoded at the LDPC encoder. An irregular LDPC code defined by $696 \times 1392$ parity check matrix as $(1392,696)$ LDPC with a code rate of $r=k / n$ generated in Matlab. Thus the LDPC encoder has $\mathrm{k}(696)$ input bits and $\mathrm{n}(1392)$ coded output bits. These coded bits are interleaved to minimize burst error and subsequently applied to the 1/2-rated (3/4-rated) convolutional encoder considering the constraint length of 7 and modulated digitally by binary phase shift keying (BPSK)/ quadrature phase shift keying (QPSK)/ quadrature amplitute modulation (QAM) at the modulator. The outputs of the modulator are complex BPSK/QPSK/QAM modulation symbols, which are passed to the Alamouti STBC encoder where the symbol values are multiplexed to the two antennas. In the OFDM block instant $(2 i), X_{1, k}$ and $X_{2, k}$ are mapped to the k-th subcarrier to be transmitted from antenna 1 and 2 . Next at the block instant $(2 i+1),-X_{2, k}^{*}$ and $X_{1, k}^{*}$ are mapped to the k-th subcarrier to be transmitted from antenna 1 and 2. The transmitted STBCOFDM symbols are given by

$$
\left(\begin{array}{l}
X_{1} \\
X_{2}
\end{array}\right) \rightarrow\left(\begin{array}{cc}
X_{1} & -X_{2}^{*} \\
X_{2} & X_{1}^{*}
\end{array}\right)
$$

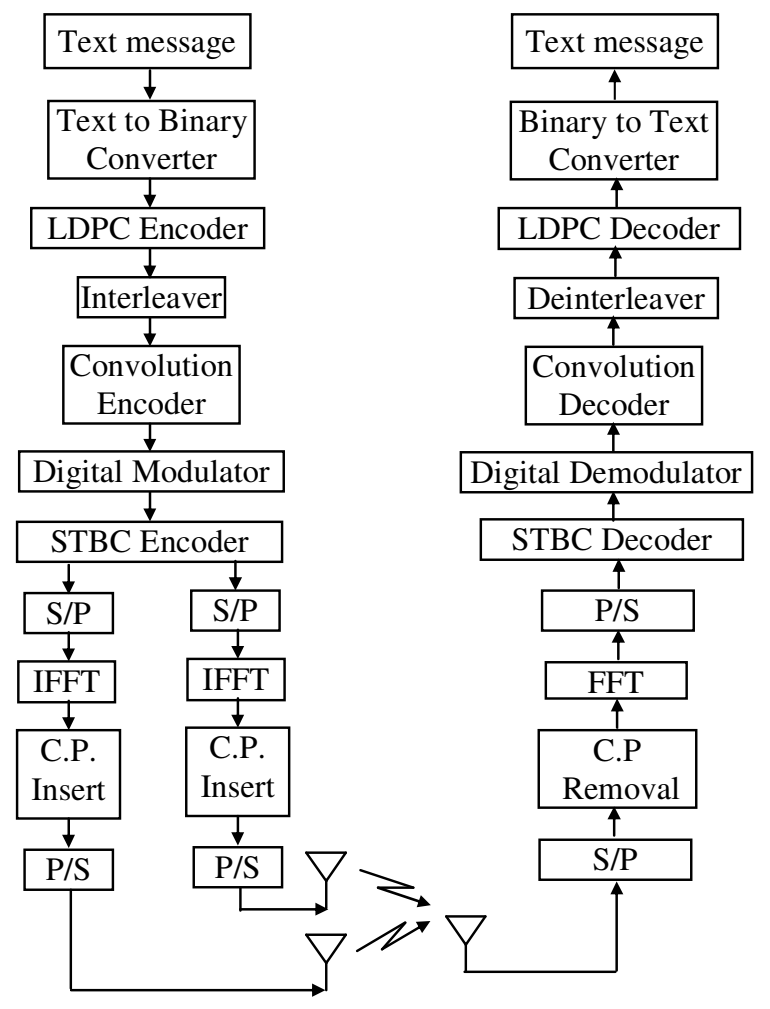

Figure 1. Block diagram of a concatenated STBC-LDPC encoded OFDM system.

At the receiver, the received time domain signals after discarding the cyclic prefix are passed through an fast Fourier transform (FFT) operation to obtain the k-th subcarrier values for symbol period $(2 i)$ and $(2 i+1)$. The signals are then fed to the Alamouti STBC decoder which 
apply maximum ratio combining (MRC) technique for providing strongest outputs. In MRC, signals from all paths are cophased and summed with optimal weighting to maximize combiner output signal to noise ratio (SNR). The outputs of the STBC decoder are subsequently demapped, convolutionally decoded, deinterleaved and then fed to the LDPC decoder to retrieve bits using an iterative sum-product algorithm. Finally the LDPC decoded binary bit stream is converted into text message.

\section{Performance Analysis}

Computer simulation using text message transmission has been performed to evaluate the BER performance of the concatenated LDPC encoded OFDM system under different modulation schemes. Figs. 2 through 5 show the BER performance of a concatenated LDPC encoded OFDM system under three types of digital modulations (BPSK, QPSK and QAM) on both AWGN and fading channels.

As seen from Figure 2 shows that the 1/2-rated convolution and LDPC encoded OFDM system outperforms at BPSK modulation and the system provides worst performance in the case of QPSK on the AWGN channel. For a typical value of energy per bit to noise ratio $\left(E_{b} / N_{0}\right)$ of $0.75 \mathrm{~dB}$, the BER values of BPSK and QPSK are 0.0007 and 0.0028 respectively which implies that the system performance with BPSK modulation is about $6.02 \mathrm{~dB}$ better than that of the system with QPSK modulation.

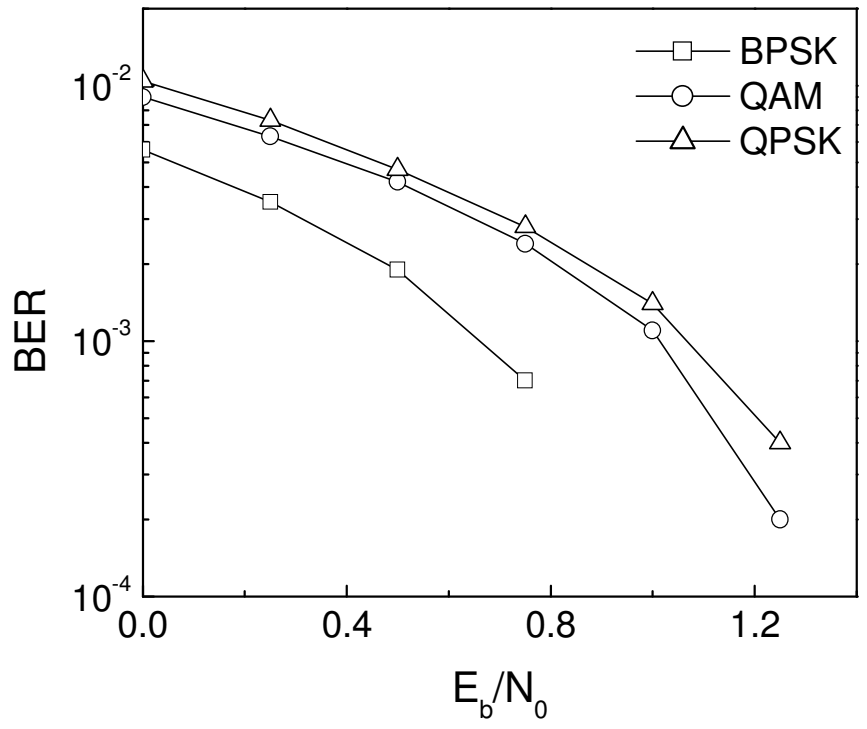

Figure 2. BER of the 1/2-rated convolution and LDPC encoded OFDM system under different modulation chemes and AWGN channel.

Figure 3 shows the BER performance of the 3/4-rated convolution and LDPC encoded OFDM system on the AWGN channel. Comparing the three represented graphical illustrations it is observable that the system performance with BPSK modulation is improved by $3.68 \mathrm{~dB}$ than that of the system with QPSK modulation for a typical value of $\left(E_{b} / N_{0}\right)$ as $0.75 \mathrm{~dB}$, where the BER values for BPSK and QPSK are 0.003 and 0.007 respectively. Comparing the graphical illustrations in Figures 2 and 3 and the BER values for a typical value of $\left(E_{b} / N_{0}\right)$ as $1.0 \mathrm{~dB}$, it 
is observable that the system performance with $1 / 2$-rated channel coding is better than that of the system with 3/4-rated channel coding.

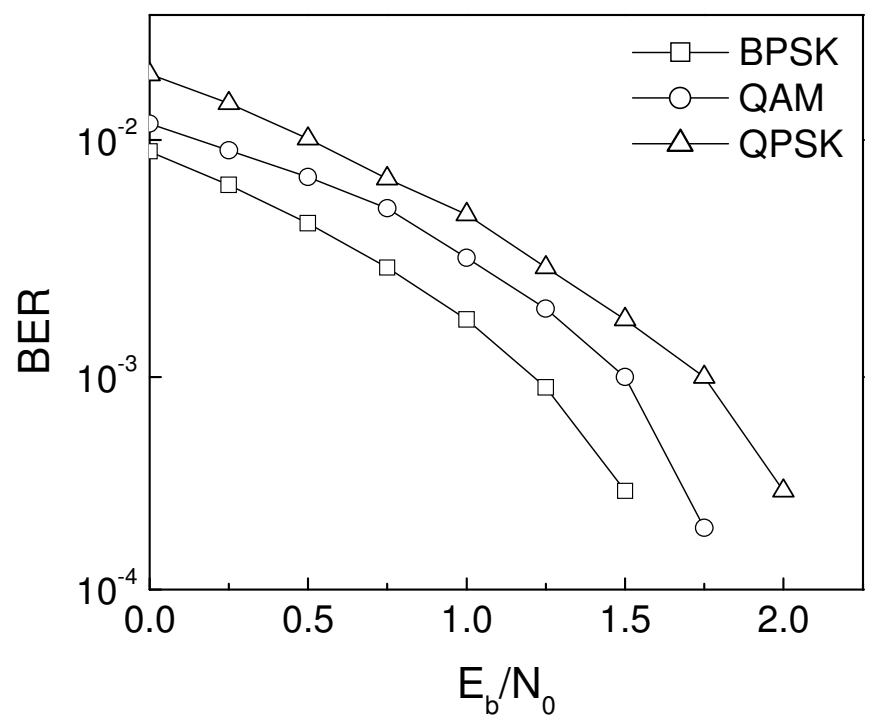

Figure 3. BER of the 3/4-rated convolution and LDPC encoded OFDM system under different modulation schemes and AWGN channel.

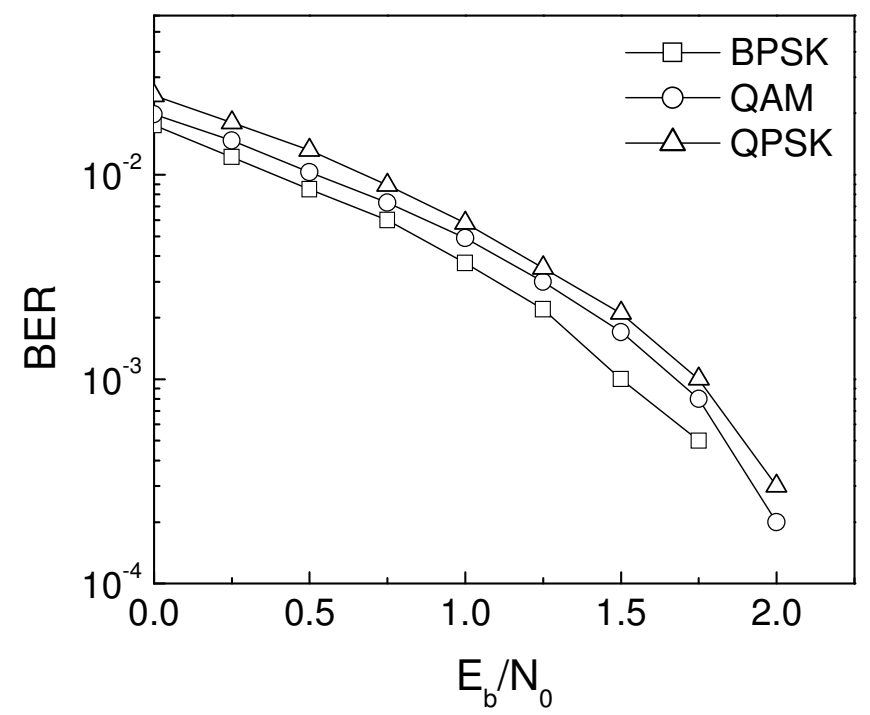

Figure 4. BER of the 1/2-rated convolution and LDPC encoded OFDM system under different modulation schemes and Rayleigh fading channel.

Figure 4 demonstrates that the BER performance of the 1/2-rated convolutional and LDPC encoded OFDM system under different digital modulations on a Raleigh fading channel degrades due to fading channel effect. The system outperforms at BPSK modulation and shows worst performance at QPSK modulation; the system with QPSK is more influenced by the Doppler frequency shift and its performance degrades. At $\left(E_{b} / N_{0}\right)$ of $1.0 \mathrm{~dB}$ (where the BER 
values for BPSK and QPSK are 0.0037 and 0.0058 respectively), the system performance is improved by $1.97 \mathrm{~dB}$ in BPSK modulation as compared with QPSK.

The BER performance of the 1/2-rated convolutional and LDPC encoded OFDM system on the Rician fading channel is shown in Figure 5. The system provides satisfactory performance with BPSK modulation. Due to fading effect, the system performance undergoes significant degradation in QPSK modulation. In comparison of the BPSK with QPSK modulation, it is found that the system performance is improved by $2.43 \mathrm{~dB}$ in the case of BPSK for a typical $\left(E_{b} / N_{0}\right.$ ) value of $1.0 \mathrm{~dB}$ (where the BER values for BPSK and QPSK are 0.002 and 0.0035 respectively).

Comparing the system performance on the Rayleigh and Rician fading channels in Figures 4 and 5, it is observable that the BER performance under Rician fading channel is better than that in Rayleigh fading channel. For a typical $\left(E_{b} / N_{0}\right)$ value of $1.0 \mathrm{~dB}$, where the BER values for BPSK under Rayleigh and Rician fading channels are 0.0037 and 0.002 respectively, which implies that the system performance is improved by $2.67 \mathrm{~dB}$.

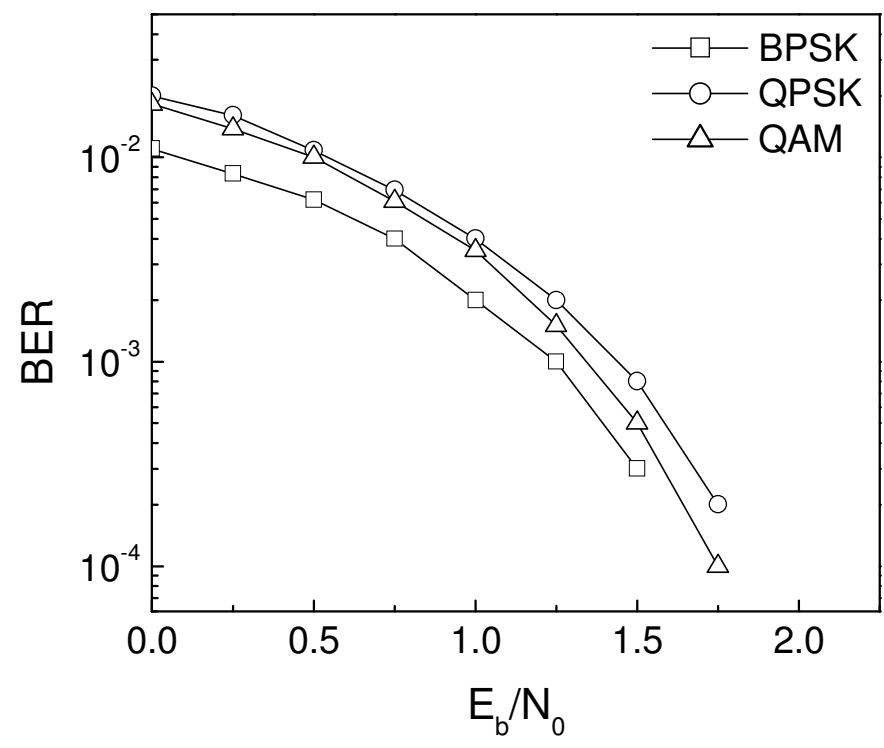

Figure 5. BER of the 1/2-rated convolution and LDPC encoded OFDM system under different modulation schemes and Rician fading channel.

Under Rayleigh fading channel environment, the transmitted and received text messages are shown in Figures 6(a) through $6(\mathrm{~d})$ for $\left(E_{b} / N_{0}\right)$ values of $0.5 \mathrm{~dB}, 1.0 \mathrm{~dB}$ and $1.5 \mathrm{~dB}$ respectively for the 1/2-rated convolutional and LDPC encoded OFDM system. The erroneous characters in the retrieved text messages are shown in bold faces (Figure 6). 
Information Technology is a very important sector in modern world. It is a computer-based education.

(a)

Information Technology is a0very important rector\$in modern world. It!is a computer-based education.

(b)

Information Technology is a very0important sector in modern world. It is a computer-fased education.

(c)

Indormation Technology is a very important sector in modern world. It is a computer-based education.

(d)

Figure 6. Transmitted text message (a) and received text messages under Rayleigh fading channel at different $\left(E_{b} / N_{0}\right)$ values of

(b) $0.5 \mathrm{~dB}$, (c) $1.0 \mathrm{~dB}$ and (d) $1.5 \mathrm{~dB}$.

\section{Conclusions}

In this paper, we have analyzed the bit error rate performance of a concatenated low density parity check encoded Alamouti-based space-time block coded Orthogonal frequency division multiplexing system under various digital modulations employing two transmit antennas and one receive antenna. It has been showed that the proposed system achieves good error rate performance under BPSK modulation technique in AWGN, Rayleigh and Rician fading channels. On the basis of the results obtained in the present simulation based study, it can be concluded that the deployment of a concatenated channel coding scheme with low-density parity-check and convolutional codes and Alamouti's transmit diversity technique in Orthogonal frequency division multiplexing based wireless communication system under BPSK modulation is very much effective in proper identification and retrieval of transmitted text message in noisy and fading environments.

\section{REFERENCES}

[1] W.C. Jakes, Jr., Ed., “Microwave Mobile Communications,” New York: Wiley, 1974.

[2] G.J. Foschini, "Layered space-time architecture for wireless communication in a fading environment when using multiple antennas,” Bell Labs Tech. J., vol.1, no.2, pp.41-59, Feb. 1996.

[3] G.J. Foschini, G.D. Golden, R.A. Valenzuela, and P.W. Wolniansky, "Simplified processing for high spectral efficiency wireless communication employing multielement arrays," IEEE J. Select. Areas Commun., vol. 17, no. 11, pp.1841-1852, Nov. 1996.

[4] S.M. Alamouti, "A simple transmit diversity technique for wireless communication," IEEE J. Select. Areas Commun., vol. 16, no. 8, pp.1451-1458, Oct. 1998.

[5] V. Tarokh, H. Jafarkhani, and A.R. Calderbank, "Space-Time block codes from orthogonal designs," IEEE Trans. Inform. Theory, vol. 45, no. 5, pp.1456-1467. Jul. 1999. 
International Journal of Computer Science, Engineering and Applications (IJCSEA) Vol.1, No.3, June 2011

[6] H. Jafarkhani, "A quasi-orthogonal space-time block code," IEEE Trans. Commun., vol. 49, pp.1-4, Jan. 2001.

[7] V. Tarokh, H. Jafarkhani, and A.R. Calderbank, "Spacetime block coding for wireless communication: Performance results," IEEE J. Select. Areas Commun., vol. 17, no. 3, pp.451-460, Mar. 1999.

[8] D. Agrawai, V. Tarokh, A. Naguib, and N. Seshadri, "Spacetime Coded OFDM for High DatarateWireless Communication Over Wideband Channels," in Proceedings of IEEE VTC 1998, (Ottawa, Canada), pp. 2232-2236, 1998.

[9] L. Hanzo, T.H. Liew and B.L. Yeap, "Turbo Coding, Turbo Equalisation and Space Time Coding for Transmission over Wireless channels,” West Sussex, England: JohnWiley and IEEE Press, 2002.

[10] C. Berrou and A. Glavieux, "Near optimum error correcting coding and decoding: Turbo-codes," IEEE. Trans. Commun., vol. 44, no. 10, pp. 1261 - 1271 Oct. 1996.

[11] H. Futaki and T. Ohtsuki, "Performance of LDPC coded OFDM Systems," IEEE VTC2001 fall, vol. 1, pp. 1696 - 1700, Oct. 2001.

[12] M. Ergen, "Mobile Broadband including WiMAX and LTE," Springer Science and Business media, LLC, USA, 2009.

[13] D.I.C. MacKay and R.M. Neal, "Near Shannon limit performance of low density parity check codes," Electron. Lett., vol. 32, no. I8, pp.1645 - 1646, Aug. 1996.

[14] M. Chiani, A. Conti and A. Ventura, "Evaluation of low-density parity check codes over block fading channels," IEEE. ICC'2000, Vol.3, pp.1183 - 1187, 2000.

[15] B. Lu, X. Wang and K.R. Narayanan, "LDPC-Based Space-Time Coded OFDM Systems Over Correlated Fading Channels: Performance Analysis and Receiver Design," IEEE Transactions on Communications, vol. 50, pp. 74-88, January 2002.

[16] J. Ha, A.N. Mody, J.H. Sung, J.R. Barry, S.W. Mclaughlin and G.L. Stüber, "LDPC coded OFDM with Alamouti/SVD diversity technique," Wireless Personal Communications, Vol. 23, pp. 183-194, 2002.

[17] M.Y. Alias, F. Guo, S.X. Ng, T.H. Liew and L. Hanzo, "LDPC and Turbo coding assisted spacetime block coded OFDM," Proc. of Vehicular Technology Conference, Vol. 4, pp. 2309-2313, 2003.

[18] J. Wu and H.-N. Lee, "Performance analysis for LDPC coded modulation in MIMO multiple access systems, "IEEE Transactions on Communications, Vol. 55, No. 7, pp. 1417-1426, July 2007.

[19] M.D. Haque, S.E. Ullah, M.M. Rahman and M. Ahmed, "BER performance analysis of a concatenated LDPC encoded OFDM system in AWGN and fading channels," J. Sci. Res., Vol. 2, No. 1, pp. 46-53, 2010.

[20] M.M. Hossain, M.M. Islam, M.S. Rahman, M.M. Islam and M.S. Islam, "BER evaluation of LDPC coded OFDM system under different modulation schemes," Inst. Eng. Tech., Vol. 1, No. 1, pp. 1-4, Jan. 2010.

\section{Authors}

M. Mesbahul Alam received the B.Sc. and M.Sc. degree in Information and Communication Engineering from University of Rajshahi, Bangladesh in 2007 and 2008, respectively. He is currently working towards his MPhil in the department of Information and Communication Engineering,University of Rajshahi, Bangladesh.

A. Z. M. Touhidul Islam received the B.Sc. and M.Sc. degree in Applied Physics and Electronics from University of Rajshahi, Bangladesh in 1997 and 1998, respectively. He has completed his PhD from 
International Journal of Computer Science, Engineering and Applications (IJCSEA) Vol.1, No.3, June 2011

Japan Advanced Institute of Science and Technology, Japan in 2009. Now he is working as an Associate Professor in the department of Information and Communication Engineering, University of Rajshahi, Bangladesh.

S. Enayet Ullah received the B.Sc. and M.Sc. degree in Applied Physics and Electronics from University of Rajshahi, Bangladesh. He has completed his $\mathrm{PhD}$ from Jahangirnagor University, Bangladesh. He is currently working as a Professor and Chairman in the department of Information and Communication Engineering, University of Rajshahi, Bangladesh. 